\title{
Instability and spatiotemporal rheochaos in a shear-thickening fluid model
}

\author{
A. Aradian* and M.E. Cates \\ School of Physics, University of Edinburgh, JCMB Kings Buildings, Edinburgh EH9 3JZ, United Kingdom.
}

(Dated: October 29, 2018)

\begin{abstract}
We model a shear-thickening fluid that combines a tendency to form inhomogeneous, shear-banded flows with a slow relaxational dynamics for fluid microstructure. The interplay between these factors gives rich dynamics, with periodic regimes (oscillating bands, travelling bands, and more complex oscillations) and spatiotemporal rheochaos. These phenomena, arising from constitutive nonlinearity not inertia, can occur even when the steady-state flow curve is monotonic. Our model also shows rheochaos in a low-dimensional truncation where sharply defined shear bands cannot form.
\end{abstract}

Complex fluids exhibit much interesting behavior under shear, due to strong couplings between mesoscopic structure and flow. Experiments show cases where, under steady external driving, an unstable flow arises, giving a time-dependent strain rate at constant imposed shear stress, or vice versa. Sustained temporal oscillations are seen in surfactant mesophases and solutions [1, 2, 3, 4, 5, 6] and polymer solutions [7], while erratic temporal responses have been found both in these and in related materials, e.g., wormlike micelles 8, 9, 10, lamellar phases 4, 5, 11 and colloids 12, 13]. There are strong indications [4, 8, 9] that these erratic signals result from a deterministic chaotic dynamics. Chaotic behavior of bulk flows at virtually zero Reynolds number (negligible inertia) must stem from nonlinearity within the rheological constitutive equation, and has been dubbed 'rheochaos' 14, 15, 16].

Such flow instabilities affect both shear-thinning $[8]$ and shear-thickening micellar materials [9]. Shear-thickening is, in itself, a widely observed but poorly understood phenomenon which affects not only micelles but, e.g., dense colloids, where there is again evidence of bulk rheological instability 12, 13. Below we study a simple model for a shear-thickening fluid at steady controlled stress, which generalises to spatially inhomogeneous flows a model first proposed in Ref. 14]. The latter was shown to give simple oscillations but not chaos (unless an unconvincing 'double memory' term was used). We find that the interplay of a very simple structural memory with constitutive nonlinearity can, as hoped by the authors of 14], give complex dynamics and rheochaos - but only if spatial heterogeneity is allowed for. Our work is related to, but different from, that of Fielding and Olmsted 16 which addresses shear-thinning fluids 17]. Also related is Ref. 15] which concerns nematic liquid crystals (which can be chaotic even without spatial heterogeneity). We show below that there are robust generic features in the rheochaos produced in these various models, which generally involve a failed attempt to create a steady shearbanded flow. (In the shear thinning case the bands have a common stress but unequal strain rates; for us, the reverse is true.) One distinctive finding of our work is rheochaos in a model where the steady state flow curve is strictly monotonic. Here (in contrast with [16]) no steady banded solution, stable or otherwise, exists. Yet an innate tendency to form bands transiently, combined with structural memory that frustrates their long-term survival, can lead to chaos. A second unexpected finding is that a low dimensional truncation of our model also gives chaos, even though sharp interfaces between shear bands are then suppressed. Thus high-order Fourier components of spatial variation are not essential to rheochaos.

Candidates for a slow structural mode include the mean length of wormlike micelles, local composition variables (e.g., colloidal volume fraction) and 'fluidity' parameters [18, 19] reflecting local microstructure, bonding state, etc.. In all cases, the time scale $\tau_{\mathrm{S}}$ is distinct from the Maxwell time $\tau_{\mathrm{M}}$ for linear stress relaxation; and in our work, we assume $\tau_{\mathrm{S}} / \tau_{\mathrm{M}} \geq 1$ (in contrast to [16]). An involvement of slowly evolving fluid structure has been clearly evidenced in some experimental cases [3, 4, 6] 6 .

The model. We assume that the shear stress $\sigma$ decouples from other stress components [20] and depends only on the rate of shear strain $\dot{\gamma}$. For a cylindrical Couette geometry with axial coordinate $z$, we consider one-dimensional inhomogeneity along this direction only (as would arise for steady shear bands in a thickening material 21]). We assume the shear flow is homogeneous within each slice of height $z$, appropriate to a lowReynolds limit; $\dot{\gamma}$ is then uniform and fixed by the imposed wall velocity. The shear stress $\sigma(z, t)$ evolves as follows, where $t$ is time and units are such that the transient elastic modulus is unity:

$$
\dot{\sigma}(z, t)=\dot{\gamma}-R(\sigma)-\lambda \int_{-\infty}^{t} \mathcal{M}\left(t-t^{\prime}\right) \sigma\left(z, t^{\prime}\right) \mathrm{d} t^{\prime}+\kappa \nabla^{2} \sigma
$$

The term $R(\sigma)=a \sigma-b \sigma^{2}+c \sigma^{3}$ corresponds to instantaneous nonlinear stress relaxation 14, with $a=1 / \tau_{\mathrm{M}}$, and $b, c$ chosen so that $\sigma(R)$ is an S-shape (see Fig. 1). This creates a tendency for the fluid to form shear bands stacked in the $z$-direction. The integral term represents a retarded relaxation of stress and/or a slow evolution of fluid structure on timescales $\tau_{\mathrm{S}}$ (see 14]). $\mathcal{M}$ is a decaying memory kernel, chosen as $\mathcal{M}(t)=\tau_{\mathrm{S}}^{-1} \exp \left(-t / \tau_{\mathrm{S}}\right)$; $\lambda>0$ governs its strength. Finally, the nonlocal term, assigning to stress a diffusivity $\kappa$, selects a unique banded flow in steady state 22]. Note that our model is closely 


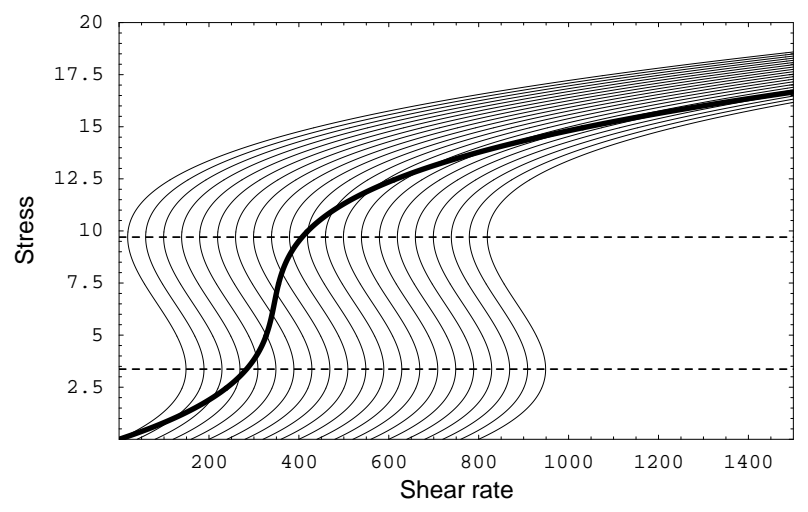

FIG. 1: Long-term flow curve $R(\sigma)=\dot{\gamma}-\lambda \sigma$ (thick line), and underlying short-term curves obeying $R(\sigma)=\dot{\gamma}-\lambda m$ (thin lines, with, from left to right, $m=0,1,2, \ldots, 20)$. The stress range between the dotted lines corresponds to the unstable region (for $\tau_{\mathrm{S}}=100$ ). Parameters: $\lambda=40, a=1 / \tau_{\mathrm{M}}=100$, $b=20, c=1.02, \kappa=0.01, H=1$.

analogous to the FitzHugh-Nagumo for neuronal activity [23], albeit with an unusual long-range coupling.

Qualitative features. We now rewrite equation (1) as an exactly equivalent differential system:

$$
\dot{\sigma}=\dot{\gamma}-R(\sigma)-\lambda m+\kappa \nabla^{2} \sigma \quad \text { and } \quad \dot{m}=-\frac{m-\sigma}{\tau_{\mathrm{S}}}
$$

The integral term in eq. (1) has become an auxiliary variable $m(z, t)$, which we call the memory. The memory encodes the delayed (structural) part of the stress relaxation, and, for our choice of $\mathcal{M}$, decays with rate $\tau_{\mathrm{S}}^{-1}$ towards the local value of the stress $\sigma(z, t)$ 24]. The homogeneous version of the present model (no $z$-dependence) shows temporal instability, leading to van der Pol type oscillations [14].

The steady-state flow curve $\sigma(\dot{\gamma})$ for the model is obtained from $\dot{\sigma}=\dot{m}=\nabla^{2} \sigma=0$, as $R(\sigma)+\lambda \sigma=\dot{\gamma}$. At each point on the curve, the memory $m$ has relaxed to the steady state stress $(m=\sigma)$. However, this relaxation involves the long timescale $\tau_{\mathrm{S}}$. At times much shorter than $\tau_{\mathrm{S}}$, the fluid will instead behave as if the memory $m$ were frozen: one has a set of 'instantaneous' flow curves $R(\sigma)+\lambda m=\dot{\gamma}$. Thus, despite the fact the steady-state flow curve is monotonic, the existence of fixed $m$ curves with an S-shape gives rise, on short times, to spatial inhomogeneity in the form of shear bands along $z$ (Fig. 1). As these bands are subjected to the local van der Pol instability referred to above, a very rich spatio-temporal dynamics arises as we now show.

Fourier-Galerkin truncation. Equations (21) were solved numerically using a spectral Galerkin truncation [25], with $\sigma(z, t)$ and $m(z, t)$ decomposed in Fourier modes $\sigma_{k}(t)$ and $m_{k}(t): \quad \sigma(z, t)=$ $\sum_{k=0}^{N-1} \sigma_{k}(t) \cos (k \pi z / H)$ etc., with $H$ the axial extent of our Couette device $(0 \leq z \leq H)$ and $N$ the order of

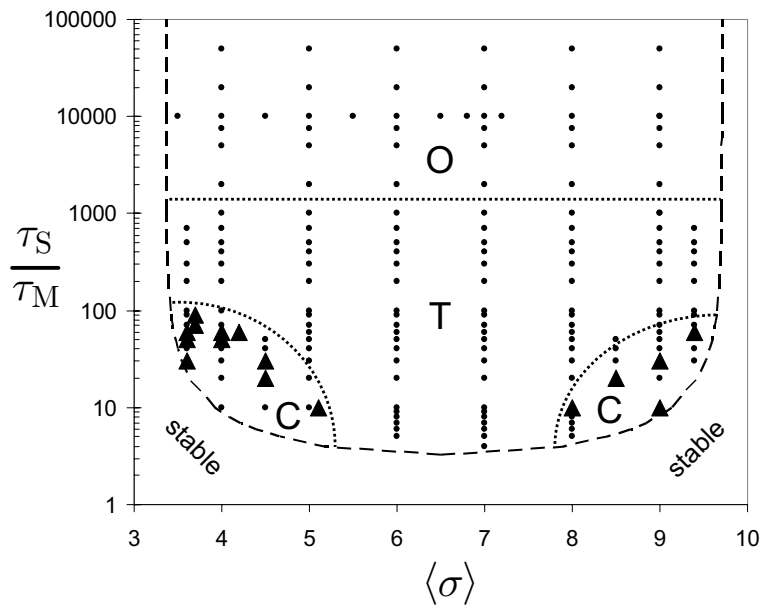

FIG. 2: Phase diagram of the model when $\tau_{\mathrm{S}}$ and $\langle\sigma\rangle$ are varied: $(\boldsymbol{\Delta})$ chaotic point, $(\bullet)$ periodic point. Three main regimes are observed: $(\mathrm{O})$ oscillating shear bands, $(\mathrm{T})$ travelling shear bands, $(\mathrm{C})$ chaotic regions. The outer dashed line is the linear stability limit, $R^{\prime}(\langle\sigma\rangle)+1 / \tau_{\mathrm{S}}=0[14,26]$. The dotted lines between regions are guides to the eye; there are no sharp transitions. The C-regions enclose all observed chaotic points, but contain internal structure with periodic and chaotic pockets. Numerical parameters as in Fig. 1.

the truncation. (Sine modes are excluded by zero stressflux boundary conditions, $\nabla \sigma=0$.) The truncation thus replaces eqs. (2) for $\sigma(z, t)$ and $m(z, t)$ by $2 N$ coupled ordinary differential equations for the modes $\sigma_{k}(t), m_{k}(t)$. The results presented below used a high-resolution truncation $(N=40)$; smaller $N(\geq 20)$ already give similar results. We work at fixed torque on the Couette, that is, at fixed value of the spatially averaged stress $\langle\sigma\rangle=\int_{0}^{H} \sigma(z, t) d z / H[26]$.

Phase diagram. In Fig. 2 we show a 'phase diagram' grouping the different types of spatiotemporal dynamics seen 27] on varying $\langle\sigma\rangle$ and $\tau_{\mathrm{S}} / \tau_{\mathrm{M}}$ (with $\tau_{\mathrm{M}}=0.01$, and remaining parameters held at the values of Fig. 1, and small amplitude noise in the initial condition). Remarkably, despite the high-dimensional dynamical system under consideration $(2 N=80)$, the phase diagram displays a simple overall structure, with three main regimes: periodic response with oscillating shear bands at extremely long $\tau_{\mathrm{S}}$; periodic response with travelling bands at long $\tau_{\mathrm{S}}$; and chaotic response at shorter $\tau_{\mathrm{S}}$ and off-centered values of $\langle\sigma\rangle$.

Oscillating shear bands. The succession of behaviors observed along the horizontal line $\tau_{\mathrm{S}} / \tau_{\mathrm{M}}=10^{4}$ in the phase diagram typifies this regime. Near the middle of the line (e.g. $\langle\sigma\rangle=7.0$ ) we find two 'flip-flopping' bands: as Fig 3-a shows, mid-cycle a low-shear band and a highshear band each span about half of the cell. Each undergoes a local van der Pol type oscillation; to keep $\langle\sigma\rangle$ fixed as prescribed, these are synchronous and each half-cycle the identity of the two bands is reversed. The time series 
(a)
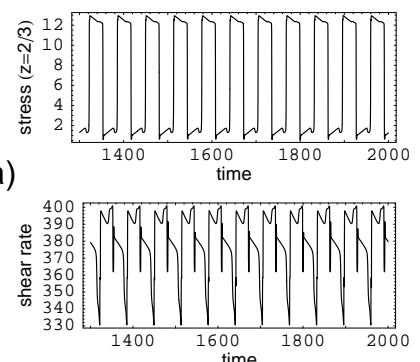

(b)
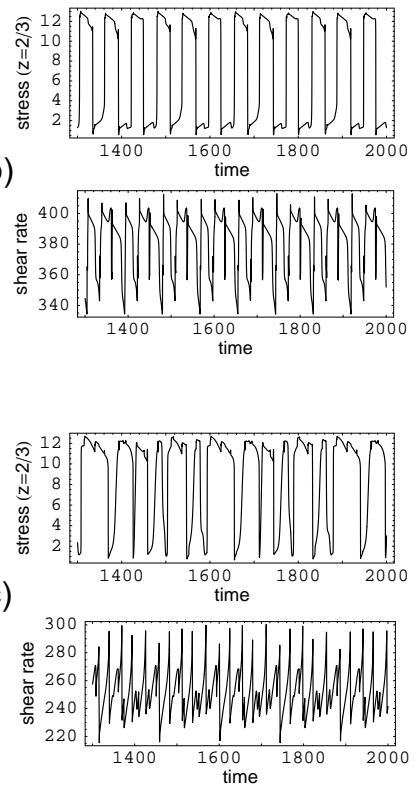
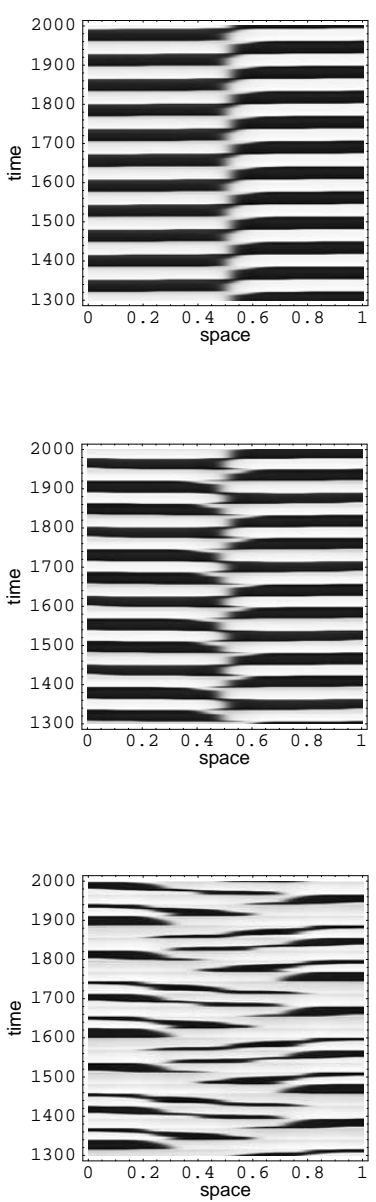

FIG. 3: Typical responses in the oscillating shear band regime, chosen along the line $\tau_{\mathrm{S}} / \tau_{\mathrm{M}}=10^{4}$ in the phase diagram. (a) Flip-flopping bands at $\langle\sigma\rangle=7$; (b) Zig-zagging interface at $\langle\sigma\rangle=7.1$; (c) Complex periodic motion at $\langle\sigma\rangle=9$. Each group presents time series of the stress $\sigma$ at $z=2 / 3$, the shear rate $\dot{\gamma}$, and a space-time plot of $\sigma(z, t)$ with $t$ vertical, $z$ horizontal (clear shades correspond to high stress, dark shades to low stress).

of the local stress (e.g., $\sigma(2 / 3, t)$; Fig 3 -a) shows a large amplitude oscillation, close to a square wave, with a flipflop period $\tau_{\mathrm{F}}$ of order $\tau_{\mathrm{S}}$. The shear rate $\dot{\gamma}$ (independent of $z$ ) accordingly shows a periodic evolution, with a more complicated waveform.

If we now raise or lower $\langle\sigma\rangle$ slightly (e.g., $\langle\sigma\rangle=7.1$, Fig. 3-b), in addition to the bands' flip-flopping, their interface adopts a zig-zag motion. (Imposition of $\langle\sigma\rangle$ now enforces unbalanced proportions of the low- and highshear bands; hence on flipping, the interface must move to and fro to maintain these proportions throughout the cycle.) The time series $\sigma(2 / 3, t)$ is slightly distorted, with period now a multiple (here three) of $\tau_{\mathrm{F}}$. Moving further towards the wings of the phase diagram, the intrinsic flipping dynamics of the bands, coupled to the global constraint on $\langle\sigma\rangle$ (e.g., $\langle\sigma\rangle=9.0$, Fig. 3-c) gives an ex-
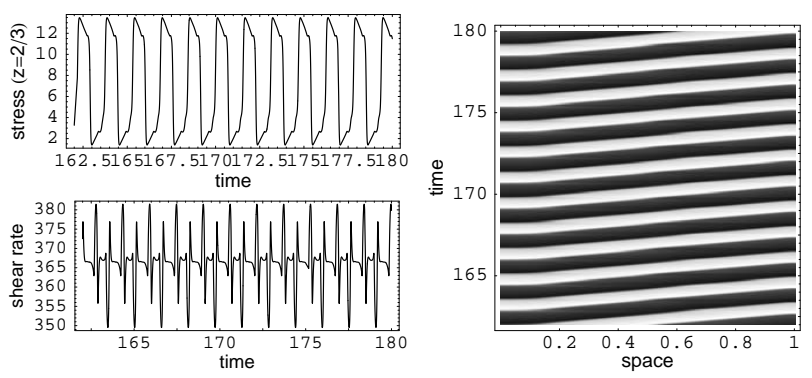

FIG. 4: Time series and space-time plot of the stress $\sigma(z, t)$ in the travelling band regime. Parameters: $\tau_{\mathrm{S}} / \tau_{\mathrm{M}}=90,\langle\sigma\rangle=7$, others as in Fig. 1.
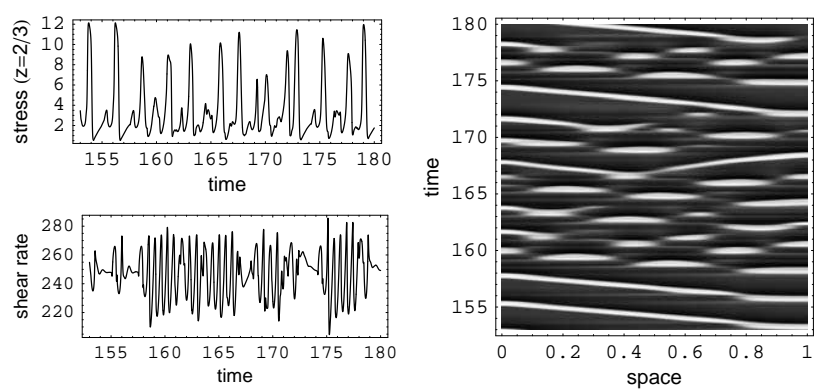

FIG. 5: Time series and space-time plot of the stress $\sigma(z, t)$ in the chaotic bands regime ('bubbly' type). Parameters: $\tau_{\mathrm{S}} / \tau_{\mathrm{M}}=90,\langle\sigma\rangle=3.6$, others as in Fig. 1 .

tremely complex motion which, remarkably, manages to remain periodic. The time series $\sigma(2 / 3, t)$ becomes very ragged and the period is a large multiple of $\tau_{\mathrm{F}}$.

Travelling shear bands. For shorter $\tau_{\mathrm{S}}\left(20 \leq \tau_{\mathrm{S}} / \tau_{\mathrm{M}} \leq\right.$ $10^{3}$ ) we find a new periodic regime in which shear bands nucleate at a boundary and then cross the system, usually one at a time, at roughly constant velocity (Fig.4). Rarely, bands can nucleate periodically from a nonboundary point [26], giving two outgoing bands in a one-dimensional analogue of the 'target patterns' seen in chemical oscillators 28]. Details here depend strongly on the initial noise. The travelling bands are kinematic waves arising from staggered phase distribution of the local van der Pol-like oscillation. Our data 26] also indicate that the band velocity decreases as $\tau_{\mathrm{S}}$ is increased. Somewhat similar band motion was reported in Ref. 16] but there the bands 'ricochet' off the walls of the container; ours disappear and renucleate at the opposite wall.

Spatiotemporal rheochaos. The third regime is chaotic and arises mainly for $10 \leq \tau_{\mathrm{S}} / \tau_{\mathrm{M}} \leq 100$, in the wings of the phase diagram. A typical dynamics in this regime (Fig. 5) comprises a 'bubbly' phase of localized, shortlived shear bands that appear and disappear erratically in the cell. (Occasionally a band survives longer and shoots across the cell.) Other types of chaotic space-time 
patterns were also found [26]: 'wiggling' travelling bands and 'defect dominated' regimes (the latter close to those found for shear thinning micelles Ref. 16]). Chaos, for each point marked in Fig. 2, was confirmed by a positive Lyapunov exponent [26].

Role of stress diffusion. The diffusivity $\kappa$ has a singular role in steady-state shear banding, selecting a unique banded flow 22. However in our work stress diffusion is, in Fourier space, a diagonal term acting mainly by damping the higher modes: small $\kappa$ increases the order $N$ required for realistic truncation, and this tends to favor chaos. We suspect that $\kappa$ also plays a role in the crossover from travelling to oscillating bands seen at $\tau_{\mathrm{S}} / \tau_{\mathrm{M}} \simeq 10^{3}$ in Fig. 2: for our parameters, this happens roughly when $\tau_{\mathrm{S}}$ becomes equal to the typical diffusion time across the Couette, $\tau_{\text {diff }}=1 / \kappa q^{2}$ (with $\left.q=\pi / H\right)$. We do find the crossover to move towards longer timescales as $\kappa$ is reduced [26].

Low-truncation limit; route to chaos. Our work, with that of 15, 16] suggests a generic interpretation of rheochaos in terms of the erratic motion of discrete band interfaces [14]. But if sharp interfaces were essential, there would be no chaos in a low-mode truncation that allows only smooth variation of $\sigma, m$ on the scale of the system. To test this, we studied the case $N=3$; with $\sigma_{0}$ and $m_{0}$ fixed by $\langle\sigma\rangle$, our dynamical variables comprise $\sigma_{1}(t)$, $\sigma_{2}(t), m_{1}(t)$ and $m_{2}(t)$. Our numerics show [26] that, although shrunk, the chaotic regions persist. Sharply resolved band interfaces are thus not essential to rheochaos. Unlike the (numerically more delicate) high-resolution truncation, the $N=3$ case allows determination of the transition to chaos as $\langle\sigma\rangle$ is varied at fixed $\tau_{\mathrm{S}}$ : we found a classical period-doubling cascade.

Concluding remarks. Our minimal model of a shearthickening fluid combines the tendency to form shear bands with structural relaxation. This combination leads to oscillatory and travelling banded solutions and to spatiotemporal chaos. Our work strongly supports a generic 'frustrated shear-banding' picture of rheochaos (e.g. 14|) as instanced in recent work on two other models [15, 16]: it seems increasingly clear that spatial inhomogeneity plays a key role in rheochaos. Intriguingly, in our model it is enough to represent this by a very low dimensional truncation of the spatial structure: transient shear bands, in the sense of sharp interfaces between layers of material of different flow history, are not essential. But, in practice, the stress diffusivity $\kappa$ usually is small and ensures rather sharp interfaces; we suspect the 'frustrated banding' picture to hold in many of the experimental realizations of rheochaos so far reported.

We thank L. Bécu, A. Colin, S. Fielding, S. Manneville, P. Olmsted, D. Roux and J.-B. Salmon for discussions. AA funded by EPSRC grant GR/R95098.
* Electronic address: A.Aradian@ed.ac.uk

[1] E. K. Wheeler, P. Fischer and G. G. Fuller, J. Non-Newt. Fluid Mech. 75, 193 (1998).

[2] Y. T. Hu, P. Boltenhagen, E. Matthys, D. J. Pine, J. Rheology 42, 1209 (1998).

[3] A.-S. Wunenburger et al., Phys. Rev. Lett. 86, 1374 (2001).

[4] J.-B. Salmon, A. Colin and D. Roux Phys. Rev. E 66, 031505 (2002).

[5] S. Manneville, J.-B. Salmon and A. Colin, Eur. Phys. J. E 13, 197 (2004).

[6] L. Courbin, P. Panizza and J.-B. Salmon, Phys. Rev. Lett. 92, 018305 (2004).

[7] L. Hilliou and D. Vlassopoulos, Ind. Eng. Chem. Res. 41, 6246 (2002).

[8] R. Bandyopadhyay, G. Basappa and A. K. Sood, Phys. Rev. Lett. 84, 2022 (2000).

[9] R. Bandyopadhyay and A. K. Sood, Europhys. Lett. 56 447 (2001).

[10] W. M. Holmes, M. R. López-González and P. T. Callaghan, Europhys. Lett. 64, 274 (2003).

[11] J.-B. Salmon, S. Manneville and A. Colin, Phys. Rev. E 68, 051504 (2003).

[12] Laun, H. M., J. Non-Newt. Fluid Mech. 54, 87-108 (1994).

[13] D. Lootens, H. Van Damme and P. Hébraud, Phys. Rev. Lett. 90, 178301 (2003).

[14] M. E. Cates, D. A. Head and A. Ajdari, Phys. Rev. E 66, 025202 (2002).

[15] B. Chakrabarti et al., Phys. Rev. Lett. 92, 055501 (2004).

[16] S. M. Fielding and P. D. Olmsted, Phys. Rev. Lett. 92, 084502 (2004).

[17] Shear thickening and thinning differ fundamentally: one cannot simply interchange stress and strain-rate variables within a dynamical model.

[18] C. Derec, A. Ajdari and F. Lequeux, Eur. Phys. J. E 4, 355 (2001).

[19] A. Aradian and M. E. Cates, in Proc. of the 3rd Int. Symp. on Slow Dynamics in Complex Systems, AIP Conference Proceedings vol. 708, M. Tokuyama and I. Oppenheim, Eds. AIP, Melville (NY), 2004.

[20] M. E. Cates, in Slow relaxations and nonequilibrium dynamics in condensed matter, Les Houches Session LXXVII, J.-L. Barrat et al., Eds. Springer, New York, 2003.

[21] J. L. Goveas and P. D. Olmsted, Eur. Phys. J. E 6, 79 (2001).

[22] C.-Y. D. Lu, P. D. Olmsted and R. C. Ball, Phys. Rev. Lett. 84, 642 (2000).

[23] J. Keener and J. Sneyd, Mathematical Physiology. Springer, New York, 1998.

[24] The form of $\mathcal{M}$ should not be important 14]; our exponential choice admits accurate and efficient numerics.

[25] J. P. Boyd, Chebyshev and Fourier Spectral Methods, Dover Publications, New York, 2000.

[26] A. Aradian and M. E. Cates, in preparation.

[27] As is expected in chaotic dynamics, there is some dependence on the numerical schemes used; see [26].

[28] I. R. Epstein and J. A. Pojman, An Introduction to Nonlinear Chemical Dynamics. Oxford University Press, Ox- 
ford, 1998. 